\title{
Lipoprotein(a) is an independent predictor of CVD
}

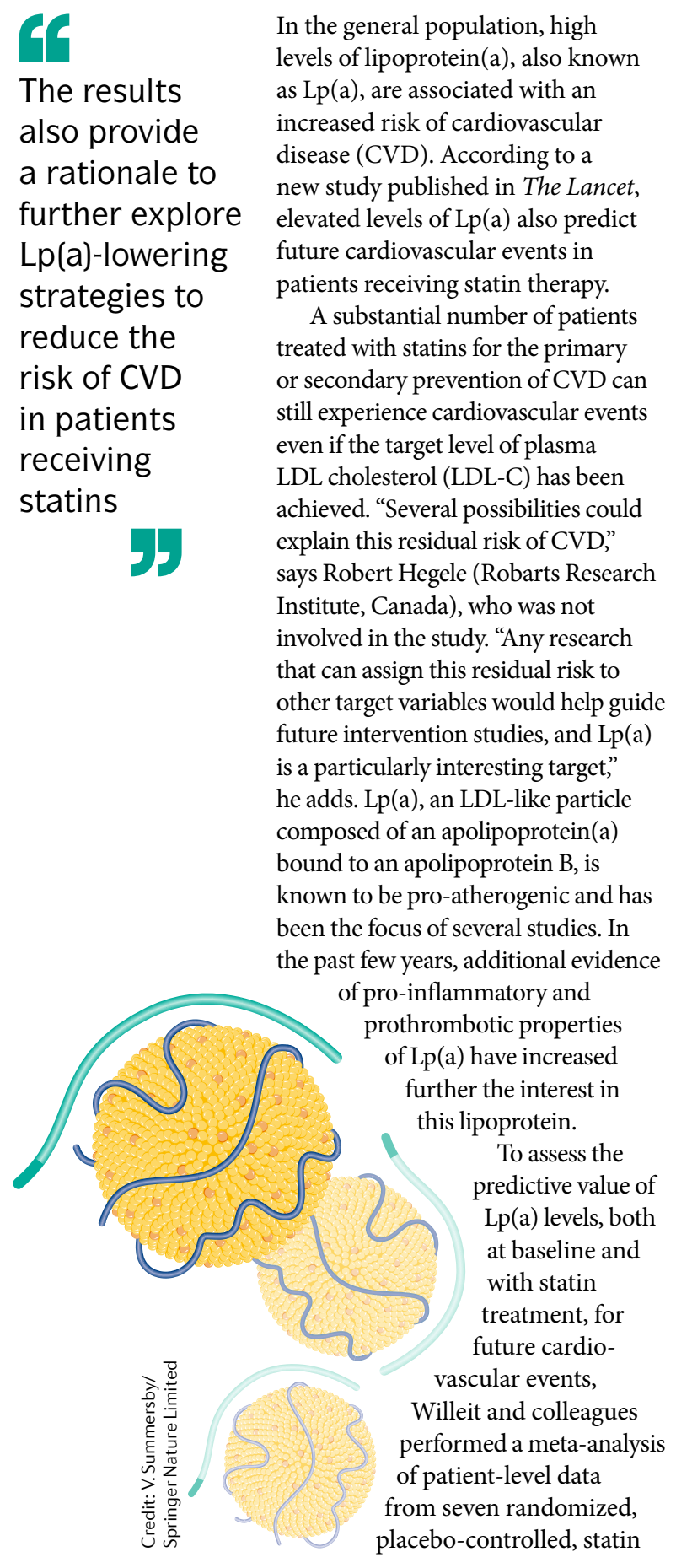

outcomes trials including 29,069 patients (14,536 allocated to receive statin therapy). The combined end point was the occurrence of fatal or nonfatal coronary heart disease, stroke, or any revascularization procedure at 3 years of follow-up.

Strengths of this meta-analysis include its design, which was well-powered to assess the risk of CVD in patients with $\mathrm{Lp}(\mathrm{a})$ levels $\geq 50 \mathrm{mg} / \mathrm{dl}$, the high number of cardiovascular events recorded and the variety of patients included, such as patients in primary or secondary prevention of CVD, patients with diabetes mellitus and patients with high LDL-C levels, which is representative of the broad population of patients who are prescribed statin therapy.

Baseline Lp(a) levels of 30-50 mg/dl (approximately $11 \%$ of the study patients) and $\geq 50 \mathrm{mg} / \mathrm{dl}$ ( $14 \%$ of the study patients) were associated with an $11 \%$ (HR 1.11, 95\% CI 1.00-1.22) and $31 \%$ (HR 1.31, 95\% CI 1.08-1.58) increased risk of cardiovascular events, respectively, compared with baseline levels of $<15 \mathrm{mg} / \mathrm{dl}$. Statin therapy reduced plasma LDL-C levels by $39 \%$ from baseline, but did not significantly change plasma Lp(a) levels. Therefore, Lp(a) levels with statin therapy had a similar association with the risk of CVD to baseline concentrations of $\mathrm{Lp}(\mathrm{a})$ : patients with $\mathrm{Lp}$ (a) levels $\geq 50 \mathrm{mg} / \mathrm{dl}$ had a $43 \%$ increased risk of cardiovascular events compared with patients with $\mathrm{Lp}$ (a) levels $<15 \mathrm{mg} / \mathrm{dl}$ (HR 1.43, 95\% CI 1.15-1.76).

The analysis also showed that high $\mathrm{Lp}(\mathrm{a})$ concentrations in plasma were associated more strongly with an elevated risk of CVD in patients receiving statins than in patients receiving placebo: compared with Lp(a) levels $<50 \mathrm{mg} / \mathrm{dl}$, Lp(a) levels $\geq 50 \mathrm{mg} / \mathrm{dl}$ were associated with a $48 \%$ (HR 1.48, 95\% CI 1.23-1.78) and $23 \%$ (HR 1.23, 95\% CI 1.04-1.45) increased risk of cardiovascular events in the groups treated with statins and placebo, respectively. Therefore, when the risk of CVD attributable to elevated LDL-C levels is reduced, the plasma level of $\mathrm{Lp}(\mathrm{a})$ becomes a strong indicator of the residual risk of CVD.

"This study dissects out the impact of increasing Lp(a) levels on cardiovascular risk, and emphasizes that $\mathrm{Lp}(\mathrm{a})$ is an independent risk predictor for CVD," comments Hegele. The results also provide a rationale to further explore $\mathrm{Lp}(\mathrm{a})$ lowering strategies to reduce the risk of CVD in patients receiving statins. To date, no drug to reduce circulating Lp(a) levels has been approved for clinical use. In phase I and phase II trials, targeting $L P A$ with antisense oligonucleotides reduced plasma Lp(a) levels by $>90 \%$. Future randomized clinical trials on the effect of these agents on cardiovascular outcomes will inform on the contribution of $\operatorname{Lp}(\mathrm{a})$ to the residual risk of CVD.

"Measuring Lp(a) levels could also be helpful clinically in risk prediction and patient stratification; it would be worthwhile to check Lp(a) levels in a patient who has suffered an event but has no traditional risk factors to explain it," concludes Hegele.

\section{Alexandra Le Bras}

ORIGINAL ARTICLE Willeit, P. et al. Baseline and on-statin treatment lipoprotein(a) levels for prediction of cardiovascular events: individual patient-data meta-analysis of statin outcome trials. Lancet 392, 1311-1320 (2018)

FURTHER READING Nordestgaard, B. G. et al. Advances in lipid-lowering therapy through gene-silencing technologies. Nat. Rev. Cardiol. 15, 261-272 (2018) 